\title{
Emission Control Policies under Uncertainty
}

\author{
GIDEON FISHELSON ${ }^{1}$ \\ Department of Economics, University of Chicago, Chicago, Illinois 60637, \\ and Tel Aviv University, Tel Aviv, Israel
}

Received March 20, 1975

\begin{abstract}
The paper deals with the problem of optimal environmental policy under uncertainty. Usually, when an environmental policy is considered, only the expected values of the parameters of the marginal benefit and marginal cost functions associated with the policy are known. Thus a relevant question is: In the presence of uncertainty, what is the optimal policy mean for achieving the environmental objective? The study addresses itself to the specific objective of improving air quality although the analysis is generally applicable. The policy means are emission taxes and emission quotas. It is shown that under uncertainty neither of these means is generally optimal, and that specific parameter values of the costs and benefits relations and their distributions determine the optimal policy for each situation.
\end{abstract}

The idea of internalizing externalities is at least as old as the first writings of Pigou. Studies advocating internalizing externalities of air and water pollution using a taxing scheme appear frequently in press nowadays $[1,2,4,5,17]$. An alternative to control via taxation is a direct regulatory approach which takes the form of emission quotas (standards). In this paper, we do not discuss the pros and cons of each regulatory system, but rather concentrate on one common problem of standards and Pigouvian tax policies. This common problem is that the optimal levels of the policies are uncertain. The uncertainty of the optimal policy level may stem from the uncertainty of the reduction of damages caused by air pollution or from the uncertainty of the costs associated with lowering pollution. The presence of uncertainty leads to the hypothesis that, in addition to economic efficiency of resource allocation, adjustment costs, and administrative costs, there is another reason that might cause one to prefer a standard to a tax, and vice versa. This paper suggests that the expected social losses due to the randomness of the parameters of the benefit and cost functions of lowering emissions are a relevant criteria. ${ }^{2}$ In the economic literature one can find relatively few discussions on the effect of price uncertainty upon social welfare $[7,10,12,19] .{ }^{3}$ However, although their uncertainty is price oriented, it is not applicable to our study. ${ }^{4}$

${ }^{1}$ Financial assistance was provided by NSF RANN (Grant No. GI 32989).

${ }^{2}$ The case for no public intervention is a priori rejected following the assumption that, whatever policy is used, social benefits would exceed social costs. The possibility of a mix of policies is not dealt with here.

${ }^{3}$ Samuelson [10] concludes the long lasting debate (started back in the forties) whether price stability is desirable from the social point of view compared to price instability as follows [10, p. 493]. "Price stability is, other things equal, a definite virtue. This is so whether one takes the view of producers or consumers or both, in the following sense. Under stable conditions of supply and demand 
In the cxamples that are worked out, the marginal cost function of emission reduction is assumed to be linear with a constant (random) slope and a random (constant) intercept. The marginal benefit function of emission reduction is assumed to be deterministic. The government is assumed to determine the policy and its level according to its knowledge of the expected value of the random parameters. Since we know (presumably) the true distribution of the random parameters, we can calculate the expected net social loss which results from each of the two policies. In this paper, we establish the conditions under which taxation is to be preferred to quotas and vice versa. It was found that when the intercept of the marginal cost curve of improving air quality is stocastic, an optimal tax would generate a lower expected social cost than would an optimal quota when the slope of the marginal cost curve exceeds that of the marginal benefit curve. When the slope of the marginal cost function is stocastic (emitters react according to the actual slope), a tax is preferable to a quota when the slope of the marginal cost is steeper (to some extent) than that of the marginal benefit curve. The required difference between the two slopes such that the tax policy is preferable to the quota policy is directly related to the variance of the random variable that affects the slope of the marginal cost curve.

\section{GENERAL BACKGROUND}

Let us start with a deterministic world. Denote the marginal social benefit function of lowering emission rate by an amount equal to $E$ by $g(E)$. The associated marginal social cost function is denoted by $h(E)$. In a quota system, the policy variable $E^{*}$ is determined such that net social benefits

$$
\int_{0}^{E^{*}}[g(E)-h(E)] d E
$$

are maximized. $E^{*}$ is accordingly the solution to the equality, $g(E)=h(E)$. In a tax system, disregarding income distribution, the optimal tax rate $t^{*}$ is defined such that $t^{*}=g\left(E^{*}\right)=h\left(E^{*}\right)$, or it can be solved directly from a maximization problem similar to (1.1) above. When the benefits and thus the marginal benefits and the costs and thus the marginal costs are stochastic, e.g., the marginals are $g(E, \alpha)$ and $h(E, \beta)$, respectively, where $\alpha$ and $\beta$ are independent random variables, $E^{*}$ is determined such that

$$
\int_{\underline{\alpha}}^{\bar{\alpha}} g(E, \alpha) f(\alpha) d \alpha=\int_{\underline{\beta}}^{\bar{\beta}} h(E, \beta) k(\beta) d \beta,
$$

where $f(\alpha)$ and $k(\beta)$ denote the density functions of the random variables. When the distributions of $\alpha$ and $\beta$ are symmetric and the functions $g(E, \alpha)$ and $h(E, \beta)$ are linear in $\alpha$ and $\beta$, the solution for $E^{*}$ under uncertainty is equal to that for a deterministic

or more basically of technology and tests, departures from price stability usually violate Pareto optimality. To the extent that producers or consumers gain, the loss to the other parties is greater than their gain."

${ }^{4}$ In the case under discussion the uncertainty is forced on by nature and we are not free to choose between stability and instability. The question to be answered is: Given that instability prevails, what is the best policy for emission control, taxes or quotas (see the answer by Oi [7])? In this sense our study is more in line with recent studies $[6,9,11]$. 


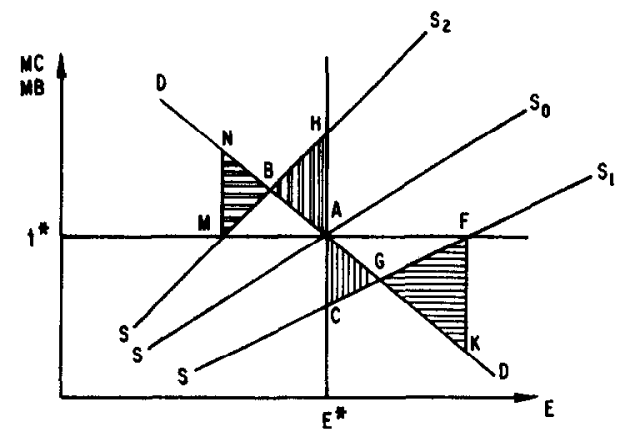

FiG. 1. Marginal costs and marginal benefits of lowering emission rates.

world, regardless of whether $g(\cdot)$ and $h(\cdot)$ are separable in their arguments or not. This is not, however, the case for the determination of the optimal tax. One can show that for the optimal tax to render $E^{*}$, where $E^{*}$ is the solution for optimal $E$, the $g(E, \alpha)$ and $h(E, \beta)$ functions should be linear and separable in $E, \alpha$, and $\beta$, respectively. Hence, they have to follow the certainty equivalent conditions (e.g., [14-16]).

In Fig. 1, the $M B$ schedule denotes the actual social marginal benefits from lowering emission rates (air quality is improved by increasing $E$ ). The $M C$ schedule denotes the actual marginal cost of lowering emissions. ${ }^{5}$ In order to solve analytically for the social losses due to uncertainty when a quota or a tax policy is imposed, we approximate the two functions in the neighborhood of the optimal solution by the linear segments,

$$
\begin{aligned}
D D: M B & =a+b E, \\
S S: M C & =c+d E .
\end{aligned}
$$

In a deterministic world, an environmental policy that results in an equilibrium different from that described by point $A$ is nonoptimal. At other equilibria (e.g., $B$ or $G$ ), social welfare is less than that in the optimal solution by an amount represented by the area of the corresponding triangles $(A B H$ or $A G C)$. Losses of welfare (e.g., the areas $A G C$ and $A B H$ ) would be generated in a deterministic world if the government is misinformed about the "true" cost function, and thus determines the standard according to $S_{1}$ (or $S_{2}$ ). Using a tax, the welfare losses due to the lack of correct information are represented by $G F K$ and $B M N$, respectively. In a static and deterministic world, one may assume that the policy maker has full and accurate information (unless the government was misinformed on purpose). This assumption weakens in a dynamic and stocastic world. The control policy is specified in terms of either a (fixed) quota or a (fixed) tax. Due to disturbances (technological progress, population growth, or random effects), the actual parameters of the marginal benefit and cost functions prevailing at each moment in the future differ from those according to which the policy was specified. In such a case, there are social losses even if the emitters react according to the parameters the policy was initially determined according to (i.e., they agree with the government). The loss due to randomness becomes more prominent when the policy is based upon some specific values of the parameters while the emitters react according to the actual (random) values prevailing at any moment. The latter is emphasized in this study.

\footnotetext{
${ }^{5}$ The analysis presented is a flow analysis. Correspondingly, both benefits (saved damages) and
} costs are flows related to the flow of emissions. 
The state of randomness is related to the parameters of the $D D$ and $S S$ functions in the neighborhood of $A$. The following four statements and assumptions incorporate the randomness into the system.

(1) Let $\bar{a}, \bar{b}, \bar{c}$, and $\bar{d}$ be the actual (random) parameters.

(2) Let $\bar{a}=a+\alpha$,

$$
\begin{aligned}
& \bar{b}=b+\beta, \\
& \bar{c}=c+\gamma, \\
& \bar{d}=d+\delta .
\end{aligned}
$$

$a, b, c$, and $d$ are known constants; $\alpha, \beta, \gamma, \delta$ are independent random variables with a known mean and variance.

(3) Government policy is specified in terms of $E$ (quota; i.e., required abatement), or in terms of $t$ (tax per unit of emission). In the latter case, the emitters react to the tax by equating $M C$ to $t$. The (fixed) levels of the policy variables are

$$
\begin{aligned}
E & =\frac{a-c}{d-b}, \\
t & =\frac{d a-b c}{d-b} .
\end{aligned}
$$

(4) The optimal emission reduction standard, $E^{*}$, and the optimal tax, $t^{*}$, are random variables

$$
\begin{aligned}
E^{*} & =\frac{\bar{a}-\bar{c}}{\bar{d}-\bar{b}} \\
t^{*} & =\frac{\bar{d} \bar{a}-\bar{b} \bar{c}}{\bar{d}-\bar{b}}
\end{aligned}
$$

The stochastic effects cause $E$ to diverge from $E^{*}$. This happens directly when an emission quota, $E$, is specified (instead of $E^{*}$ ), or, indirectly when a tax, $t$, is specified instead of $t^{*}$, in which case the emitters react as indicated above.

The observed reduction in the emission rate is denoted by $E^{\prime}$. The social costs (with respect to the optimum) are therefore ( $X$ is the integration variable)

$$
\begin{aligned}
S C & =\int_{E^{\prime}}^{E^{*}}[(\bar{a}-\bar{c})+(\bar{b}-\bar{d}) X] d X \\
& =(\bar{a}-\bar{c})\left(E^{*}-E^{\prime}\right)+\frac{\bar{b}-\bar{d}}{2}\left[\left(E^{*}\right)^{2}-\left(E^{\prime}\right)^{2}\right] .
\end{aligned}
$$

\section{GOVERNMENT-EMITTERS DISAGREE (OR GOVERNMENT IS "MISINFORMED”)}

When analyzing either the case of certainty or uncertainty, the situation of different viewpoints of government (public) and polluters with regard to the parameters of the marginal cost function must be considered. A disagreement might exist, for example, due to a time lag between the policy legislation and policy application. Accordingly, government policy is based upon $a, b, c$, and $d$, while the "actual" parameters, ac- 
cording to which the emitters reacl, are $c+\gamma(=\bar{c})$ and $d+\delta(=\bar{d})$. As before, let $\alpha, \beta, \gamma$, and $\delta$ be random variables. The government sets the emission reduction quota to equal

or, the emission tax to equal

$$
E=\frac{a-c}{d-b}
$$

$$
t=\frac{d a-b c}{d-b} .
$$

The rigidity of a policy variable has a different effect on the social cost depending on the policy tool. Generally, a tax system allows flexibility with regard to the quantity to be abated, while a quota sets a lower limit. Thus, on initial grounds, a tax seems to be preferred to a quota. The analysis below refutes this simple intuitive argument.

The situation for $\alpha$ being the random variable is presented in Fig. 2 for a two-point distribution. The social loss from a (fixed) quota $E$ is approximated by the triangles $A B C$ and $B G F$ whose area is random when $\alpha$ is random. The detailed analysis of this case and the one in which $\beta$ is a random variable is not presented here. The conclusions (for a quota versus a tax system) are identical to those reached for the case where the emitters react according to the same parameters the government policy is based upon but both emitters and government are wrong. The more relevant and interesting cases are those where government sets the policy variable according to some specific values of the parameters of the marginal cost function while the emitters react according to the actual parameters.

First, let the intercept of the marginal cost function be stocastic. Government's quota is $E$, while the optimal (random) quota is $E^{*}\left(E_{1}{ }^{*}\right.$ or $E_{2}{ }^{*}$ in Figs. 3a and $3 \mathrm{~b}$ ). Obviously, $E$ is the observed reduction in emissions. Since the optimal reduction is $E^{*}$,

$$
E_{i}^{*}=\frac{a-(c+\gamma)}{d-b}, \quad \text { for } i=1,2
$$

The expected social loss is

$$
E\left\{S C_{1}^{1}\right\}=\frac{1}{2(d-b)} \sigma_{\gamma}^{2},
$$

which corresponds to the expected area of triangels $R K M$ and $N K L$. Now, let the government impose a tax $t$. The optimal reduction in emission is as before (Eq. 2.3),

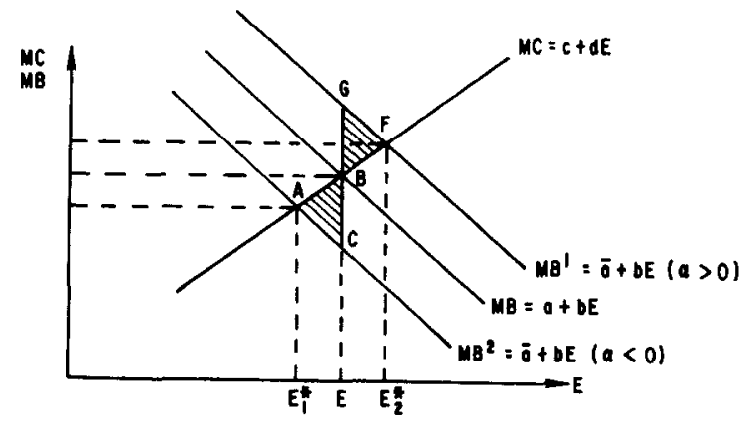

Frg. 2. Social costs of a stocastic "demand" function. The triangles $A B C$ and $B G F$ are identical for a tax and a quota. 
(o)

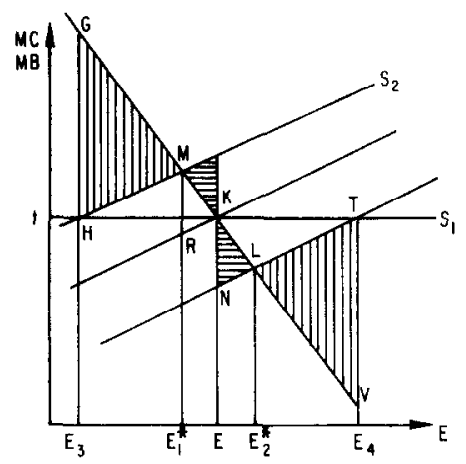

(b)

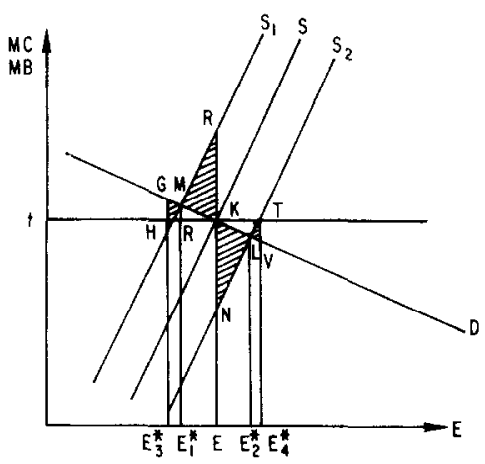

Fig. 3. Social costs (the shaded areas) of a stocastic marginal cost.

while the actual reduction by the polluters would be $E_{3}$ (or $E_{4}$ ),

Hence,

$$
E_{i}=\frac{t-(c+\gamma)}{d}, \quad \text { for } i=3,4
$$

$$
E_{i}=\left[\frac{d a-b c}{(d-b)}-(c+\gamma)\right] / d=\frac{a-(c+\gamma)}{(d-b)}+\frac{b \gamma}{d(d-b)} .
$$

It is obvious that the $E$ observed when imposing a tax does not equal $E$ imposed as a quota. The difference is $b \gamma / d(d-b)$. Utilizing Eq. (1.7) one finds that when a tax is imposed the expected social costs because of the randomness of the intercept of the air quality "supply function" (according to which the emitter behaves), is

$$
E\left[S C_{1}^{2}\right]=\left(\frac{b}{d}\right)^{2} \cdot \frac{1}{2(d-b)} \sigma_{r}^{2} .
$$

Hence, the decision on a tax system versus a quota system depends on the ratio $|b| / d$. When the marginal benefit of air quality is steeper than the corresponding marginal cost, the quota system results in lower expected costs than the tax system. The tax system has to be favored when the marginal cost curve is steeper than the marginal benefit curve. ${ }^{6}$

The next case to be analyzed is when the slope of the marginal cost curve in the neighborhood of the equilibrium is random. The quota set by the government is as before,

$$
E=\frac{a-c}{d-b}
$$

( The government sets the quota and tax using the expected values of the parameters). The optimal (random) quota is

$$
E^{*}=\frac{a-c}{(d-\delta)-b} .
$$

' Those results are identical to Upton's $[18]$ conclusions. 
The social costs due to the divergence between $E$ and $E^{*}$ are (Figs. $3 a$ and $3 b$, triangles $R K M$ and $N K L$ )

$$
S C_{\perp}^{2}=\frac{(a-c)^{2}}{2(d+\delta-b)(d-b)^{2}} \delta^{2} .
$$

The expected value of these costs is given in Appendix A.

Under a tax policy, the tax imposed is

$$
t=\frac{d a-c b}{d-b}
$$

Following their (actual) marginal cost function, the emitters reduce emission rate by

$$
E=\frac{t-c}{d+\delta}=\frac{d(a-c)}{(d-b)(d+\delta)} .
$$

The optimal reduction is (as above)

$$
E^{*}=\frac{a-c}{(d+\delta)-b} .
$$

The social costs associated with the difference between actual reduction and optimal reduction are (Figs. $3 \mathrm{a}$ and $3 \mathrm{~b}$, triangles $M G H$ and $L T V$ )

$$
S C_{2}{ }^{2}=\frac{(a-c)^{2}}{2(d+\delta-b)(d-b)^{2}(d+\delta)^{2}} b^{2} \delta^{2} .
$$

Note the similarity between $S C_{1}{ }^{2}$ and $S C_{2}{ }^{2} . S C_{2}{ }^{2}$ is multiplied by an additional term $b^{2} /(d-\delta)^{2}$, as compared to $S C_{1}{ }^{2}$. Recall that in the case where the intercept of the marginal cost curve was the random variable, $S C_{2}{ }^{1}$ was multiplied by $(b / d)^{2}$ as compared to $S C_{1}{ }^{1}$. The expected value of $S C_{2}{ }^{2}$ is given in Appendix B. A comparison between $E\left[S_{2}{ }^{2}\right]$ and $E\left[S C_{1}{ }^{2}\right]$ is presented below. The difference between the expected social costs of a tax system versus a quota system equals the expected value of the difference.

$$
\begin{aligned}
& E\left[S C_{2}{ }^{2}\right]-E\left[S C_{1}{ }^{2}\right]=E\left[S C_{2}{ }^{2}-S C_{1}{ }^{2}\right] \\
& =\int_{\delta=\delta_{1}}^{\delta_{2}}\left(\frac{-(a-c)^{2} b^{2} \delta^{2}}{2(d+\delta-b)(d-b)^{2}(d+\delta)^{2}}-\frac{(a-c)^{2} \delta^{2}}{2(d+\delta-b)(d-b)^{2}}\right) f(\delta) D \delta \\
& \quad=\frac{(a-c)^{2}}{2(d-b)^{2}} \int_{\delta=\delta_{1}}^{\delta_{2}} \frac{\delta^{2}}{(d+\delta-b)}\left(\frac{b^{2}}{(d+\delta)^{2}}\right) f(\delta) D \delta, \quad
\end{aligned}
$$

where $f(\delta)$ is the density function of the random variable $\delta$.

Assume that $|\delta|<d$; i.e., the $M C$ curve never becomes infinitely elastic (and obviously not negatively sloped). A sufficient condition for the integral to be always positive is: $b^{2}>(d+\delta)^{2}$, or $|b|>d+\delta$. Hence, if for the largest positive level of the random variable that affects the slope of marginal cost curve, the slope of the marginal benefit curve is steeper, the tax system would generate an expected social loss that is larger than that rendered by a quota system. The other extreme case is defined for 
$d+\delta>|b|$, for any level of $\delta$. If $d+\delta>|b|$ for any level of $\delta$, then a quota system would generate an expected social cost that is larger than that generated by a tax system. For a changing world which stays within boundaries, i.e., for some $\delta$, $d+\delta<|b|$ while for other $\delta, d+\delta\rangle|b|$, there is no definite general answer with regard to the optimal policy. The answer depends on the specific relevant parameters.

The above discussion requires one reservation. The quota-tax comparison is carried out using the certainty equivalent assumption for an optimal quota and optimal tax, although the certainty equivalent principle is not applicable for the determination of the optimal tax (we compare controls which are not optimal to start with). ${ }^{7}$

\section{CONCLUSIONS}

In the analysis, we showed that an optimal behavior of the emitters (according to the actual marginal cost parameters) generates differences in the expected social losses from a quota policy and a tax policy. These differences depend upon the relative slopes of the marginal benefit and marginal cost functions. It can also be shown that the randomness of the parameters of the marginal benefit function is irrelevant to the decision on the policy mean since the expected social losses from a quota and a tax policy are identical. The moral is obvious. The main effort is to be put into the study of the pollution abatement activities and costs, before resorting to any specific policy.

In order to avoid excess generalization of the conclusions, the reader is reminded that the analysis and results hold only under the assumption of linear marginal benefits and marginal cost functions. Once nonlinearity is introduced (e.g., marginal costs increase at an increasing rate or marginal benefits decline at an increasing rate) the conclusions cannot be carried over without a reexamination of the expected social costs due to uncertainty.

\section{APPENDIX A: THE EXPECTED VALUE OF $S C_{1}{ }^{2}$}

Following the definition of an expected value, one gets ( $D \delta$ stands for the mathematical notation $d \delta$ where $\delta$ is the variable over which the integration is done)

$$
E\left[S C_{1}{ }^{2}\right]=\frac{(a-c)^{2}}{2(d-b)^{2}} \int_{\underline{\delta}}^{\delta} \frac{\delta^{2}}{d+\delta-b} f(\delta) D \delta .
$$

Let $\delta$ be distributed uniformly over the range $-k d$ to $k d$. Hence, $E[\delta]=0$; Var $(\delta)$ $=k^{2} \delta^{2} / 3$ and $f(\delta)=1 /(2 k d)$. Introducing this information into (A1) renders

$$
E\left[S C_{1}^{2}\right]=\frac{(a-c)^{2}}{4 k d(d-b)^{2}} \int_{-k d}^{k d} \frac{\delta^{2}}{d+\delta-b} D \delta .
$$

The solution is (following [13, p. 397, Eq. 33])

$$
\begin{aligned}
& E\left[S C_{1}^{2}\right]=\frac{(a-c)^{2}}{4 k d(d-b)^{2}}\left[\frac{1}{2}(d-b+\delta)^{2}-2(d-b)(d-b+\delta)\right. \\
& \left.\quad+(d-b)^{2} \log (d-b+\delta)\right]_{-k d}^{k d}=\frac{(a-c)^{2}}{4 k d}\left[\log \frac{d(1+k)-b}{d(1-k)-b}-\frac{2 k d}{(d-b)}\right]
\end{aligned}
$$

${ }^{7}$ This topic is further elaborated upon in an unpublished manuscript by Fishelson and Flatters [3]. 


\section{APPENDIX B: THE SOLUTION FOR $E\left[S C_{2}{ }^{2}\right]$}

Following the procedure and assumptions presented in Appendix A and [13, p. 399, Eq. 56], we get the following solution.

$$
\begin{aligned}
E\left[S C_{2}{ }^{2}\right]=\frac{(a-c)^{2}}{4(d-b)^{2} k d}\left[\frac{b}{(1-k)^{2}}+(d-b)^{2} \log \frac{d(1+k)-b}{d(1-k)-b}\right. & \\
& \left.+d^{2} \log \frac{(1+k)}{(1-k)}\right] .
\end{aligned}
$$

\section{REFERENCES}

1. W. J. Baumol and W. E. Oates, The use of standards and prices for protection of environment, The Swedish Journal of Economics 73, 42-54 (1971).

2. W. J. Baumol, The instruments for environmental policy, Paper presented at the Conference on Economics of the Environment, NBER (November 1972).

3. G. Fishelson and F. Flatters, The non equivalence of tariffs and quotas under uncertainty, unpublished paper, University of Chicago (December 1974).

4. J. E. Hass, Optimal taxing for the abatement of water pollution, Water Resource Research 6, 353-365 (1970).

5. Margareta Johanesson, Dynamic aspects on the use of prices for protecting the environment, The Swedish Journal of Economics 74, 286-291 (1972).

6. J. J. McCall, Competitive production for constant risk utility functions, Rev. Econ. Stud. 4, 417420 (1967).

7. Walter Y. Oi, The desirability of price instability under perfect competition, Econometrica 29, 58-64 (1961).

8. Walter Y. Oi, The consumer does benefit from feasible price stability: A comment, Quarterly Journal of Economics 86, 494-498 (1972).

9. William Poole, Optimal choice of monetary policy instruments in a simple stochastic macro model, Quarterly Journal of Economics 84, 197-216 (1970).

10. P. A. Samuelson, The consumer does benefit from feasible price instability, Quarterly Journal of Economics 86, 476-493 (1972).

11. A. Sandmo, On the theory of the competitive firm under price uncertainty, American Economic Review 61, 65-73 (1971).

12. Richard Schmalensee, Option demand and consumer's surplus: Valuing price changes under uncertainty, American Economic Review 62, 813-824 (1972).

13. S. M. Selby, "Standard Mathematical Tables," 18th Ed. The Chemical Rubber Company, Cleveland, Ohio (1970).

14. H. A. Simon, Dynamic programming under uncertainty with quadratic criterion functions, Econometrica 24, 74-81 (1956).

15. H. Theil, Econometric models and welfare maximization, Weltwirtschaftliches Arciv 72, 60-83 (1954).

16. H. Theil, A note on certainty equivalence in dynamic planning, Econometrica 25, 346-349 (1957).

17. C. Upton, Optimal taxing of water pollution, Water Resources Research 4 (October 1968).

18. C. Upton, The allocation of pollution rights, Urban Economics Report No. 59, University of Chicago (1971).

19. F. V. Waugh, Consumer aspects of price instability, Econometrica 34, 504-508 (1966). 